$20^{\text {th }}$ European Symposium on Computer Aided Process Engineering - ESCAPE20

S. Pierucci and G. Buzzi Ferraris (Editors)

(C) 2010 Elsevier B.V. All rights reserved.

\title{
Computer Aided Design of Plate Heat Exchangers
}

\author{
Olga Arsenyeva ${ }^{\mathrm{a}}$, Leonid Tovazhnyansky ${ }^{\mathrm{a}}$, Petro Kapustenko ${ }^{\mathrm{a}}$, Gena Khavin ${ }^{\mathrm{b}}$ \\ ${ }^{a}$ National Technical University "Kharkiv Polytechnic Institute", \\ 21 Frunze Str., 61002, Kharkiv, Ukraine, kap@kpi.kharkov.ua \\ ${ }^{\mathrm{b}}$ AO SODRUGESTVO-T, 2 Krsnoznamenny Per., 61002, Kharkiv, Ukraine, \\ sodrut@gmail.com
}

\begin{abstract}
The computer aided design of plate heat exchanger with mixed grouping of plates is considered. It is formulated as the mathematical problem of finding the minimal value for implicit nonlinear discrete/continues objective function with inequality constraints. The optimizing variables include the number of passes for both streams, the numbers of plates with different corrugation geometries in each pass, the plate type and its size. To estimate the value of objective function in a space of optimizing variables the mathematical model of plate heat exchanger is developed. To account for thermal and hydraulic performance of channels between plates with different geometrical forms of corrugations, the exponents and coefficients in formulas for heat transfer coefficients and friction factors calculation are used as model parameters. The procedure and software for numerical experiment to identify model parameters by comparing the calculation results with those obtained with free available in web computer programs of plate manufacturers is developed. The sets of such parameters are obtained for a number of industrially manufactured plates. The described approach is implemented as software for plate heat exchangers calculation.
\end{abstract}

Keywords: Plate Heat Exchanger, Design, Mathematical Model, Model Parameters

\section{Introduction}

Plate heat exchangers (PHEs) are one of the most efficient types of heat transfer equipment. The principles of their construction and design methods are sufficiently well described elsewhere, see e.g. Hesselgreaves (2001), Wang, Sunden and Manglik (2007), Tovazshnyansky et al (2004). This equipment is much more compact and requires much less material for heat transfer surface production, much smaller footprint, than conventional shell and tubes units. PHEs have a number of advantages over shell and tube heat exchangers, such as compactness, low total cost, less fouling, flexibility in changing the heat transfer surface area, accessibility. Due to differences in construction principles from conventional shell and tube heat exchangers, PHEs require substantially different methods of thermal and hydraulic design. Such methods should be based on accurate enough mathematical models.

\section{Mathematical model of PHE}

The PHE consists of a set of corrugated heat transfer plates clamped together between fixed and moving frame plates and tightened by tightening bolts, see e.g Hesselgreaves (2001). The plates are equipped with the system of sealing gaskets, which are also separate the streams and organizing their distribution over the inter plate channels. In multi-pass PHE plates are arranged in such way, that they are forming groups of parallel channels. Such group is corresponding to one pass and the stream is going consequently 
through the passes. The temperature distributions in passes are different, and in different groups of channels both counter-current and co-current flows may occur.

The mathematical model of PHE was presented by Arsenyeva et al.(2009). The PHE is regarded as a system of one-pass blocks of plates. The conditions for all channels in one such block are equal. The total number of blocks is $n_{b}=X_{1} X_{2}$ and the number of heat transfer units in one block, counted for hot stream:

$$
N T U_{b}=U_{b} \cdot F_{b} \cdot X_{2} /\left(G_{1} c_{1}\right)
$$

where $U_{b}$ - overall heat transfer coefficient in block, $\mathrm{W} /\left(\mathrm{m}^{2} \mathrm{~K}\right) ; \mathrm{G}_{1}$ - mass flow rate of hot stream, $\mathrm{kg} / \mathrm{s} ; \mathrm{c}_{1}-$ specific heat of hot stream, $\mathrm{J} /(\mathrm{kg} \cdot \mathrm{K}) ; \mathrm{X}_{1}$ and $\mathrm{X}_{2}-$ the number of passes for hot and cold stream, respectively.

When $\mathrm{G}_{1} \mathrm{c}_{1} / \mathrm{X}_{2}<\mathrm{G}_{2} \mathrm{c}_{2} / \mathrm{X}_{1}$ block heat exchange effectiveness $\varepsilon_{b}$ for counter current flow:

$$
\varepsilon_{b}=\left[1-\exp \left(N T U_{b} \cdot R_{b}-N T U_{b}\right)\right] /\left[1-R_{b} \cdot \exp \left(N T U_{b} \cdot R_{b}-N T U_{b}\right)\right]
$$

where $R_{b}=G_{1} \cdot C_{1} \cdot X_{1} /\left(G_{2} \cdot c_{2} \cdot X_{2}\right)$ - the ratio of heat capacities of streams going trough block; $G_{2}$ and $c_{2}$ mass flow rate $[\mathrm{kg} / \mathrm{s}]$ and specific heat $[\mathrm{J} /(\mathrm{kg} \cdot \mathrm{K})]$ of cold stream.

If $\mathrm{R}_{\mathrm{b}}=1$, then $\varepsilon_{b}=N T U_{b} /\left(1+N T U_{b}\right)$.

In case of co-current flow directions

$$
\varepsilon_{b}=\left[1-\exp \left(-N T U_{b} \cdot R_{b}-N T U_{b}\right)\right] /\left(1+R_{b}\right)
$$

On the other hand the heat exchange effectiveness of block $i: \varepsilon_{b i}=\delta t_{1 i} / \Delta t_{i}$,

where $\delta t_{1 i}$ - temperature drop in block $i ; \Delta t_{i}$ - the temperature difference of streams entering block $i$. The temperature change of cold stream: $\delta t_{2 i}=\delta t_{1 i} \cdot R_{b}$

These relations can be regarded as mathematical model of block, which describes the dependence of temperature changes from characterising block values of $F_{b}$ and $U_{b}$.

For every block we can write the equation which describes the link of temperature change in this block to temperature changes in all other blocks of PHE.

For any number of passes such system presented in matrix form:

$[Z]\left[\delta t_{i}\right]=\left[\varepsilon_{b i} \Delta\right]$

where $\left[\delta t_{i}\right]$ - vector-column of temperature drops in blocks; $\left[\varepsilon_{b i} \Delta\right]$ - vector-column of the right hand parts of the system; $[Z]$ - matrix of system coefficients.

The numerical solution of linear algebraic equations system (7) easily made on PC. After that the total temperatures change in PHE calculated as:

$\delta t_{\Sigma 1}=\sum_{i=1}^{X_{1}}\left(\frac{1}{X_{1}} \sum_{i i=1}^{X_{2}} \delta t_{(i-1) X_{2}+i i}\right) ; \quad \delta t_{\Sigma 2}=\frac{\left(G_{1} c_{1}\right)}{\left(G_{2} c_{2}\right)} \delta t_{\Sigma 1}$.

The total heat load of PHE:

$Q=\delta t_{\Sigma 1} \cdot G_{1} \cdot c_{1}$,

This system should be accompanied by equations for calculation of overall heat transfer coefficient $U, \mathrm{~W} /\left(\mathrm{m}^{2} \mathrm{~K}\right)$, as below.

$U=1 /\left(1 / \alpha_{1}+1 / \alpha_{2}+\delta_{w} / \lambda_{w}+R_{f}\right)$ 
where $\alpha_{1}, \alpha_{2}$ - film heat transfer coefficients for hot and cold streams, respectively, $\mathrm{W} /\left(\mathrm{m}^{2} \mathrm{~K}\right) ; \delta_{w}$ - the wall thickness, $\mathrm{m} ; \lambda_{w}$ - heat conductivity of the wall material, $\mathrm{W} /(\mathrm{mK}) ; R_{f}=R_{f 1}+R_{f 2}$ - the sum of fouling thermal resistances for streams, $\mathrm{m}^{2} \mathrm{~K} / \mathrm{W}$.

For plate heat exchangers the film heat transfer coefficients are usually calculated by empirical correlations:

$N u=f(\operatorname{Re}, \operatorname{Pr})=A^{*} \operatorname{Re}^{n} \operatorname{Pr}^{0.4}\left(\mu / \mu_{w}\right)^{0.14}$

Here $\mu$ and $\mu_{w}$ dynamic viscosity at stream and at wall temperatures, respectively;

Nusselt number is:

The streams velocities are calculated as

Where $g$ is flowrate of stream through one channel, $\mathrm{kg} / \mathrm{s}$.

The pressure drop in one PHE channel

$\Delta p=\zeta \cdot\left(L_{p} / d_{e}\right) \cdot \rho \cdot w^{2} / 2$,

where $L_{p}$-effective plate length; $\zeta$ - friction factor, which is usually determined by empirical correlations of following form:

$\zeta=B / \operatorname{Re}^{m}$

For multi pass PHE the pressure drop in one pass multiplied by number of passes $\mathrm{X}$.

In modern PHEs plates of one type are usually made with two different corrugation angles, that can form three different channels, when assembled in PHE. Plates of $\mathrm{H}$ type have corrugations with bigger angles (about $60^{\circ}$ ) that form the $\mathrm{H}$ channels with higher efficiency of heat transfer and hydraulic resistance. Plates of $\mathrm{L}$ type have a lower angle (about $30^{\circ}$ ) and form the $\mathrm{L}$ channels with lower heat transfer and hydraulic resistance. Combined, these plates form channels $\mathrm{MH}$ or ML with intermediate characteristics. Such principle of design enables to change thermal and hydraulic performance of plates pack with the level of discreteness equal to one plate in a pack.

In one PHE two groups of channels are usually used. One is of higher hydraulic resistance and heat transfer (x-channel), another of lower characteristics (y-channel). When the stream is going through set of such channels, the temperature changes in different channels are differ. After mixing in collector part of PHE block, the temperature is determined by heat balance. Then the heat exchange effectiveness of plates block with different channels:

$\varepsilon_{b}=\left(g_{x} \cdot n_{x} \cdot \varepsilon_{x}+g_{y} \cdot n_{y} \cdot \varepsilon_{y}\right) /\left(g_{x} \cdot n_{x}+g_{y} \cdot n_{y}\right)$,

where $n_{x}$ and $n_{y}$ are the numbers of $\mathrm{x}$ and $\mathrm{y}$ channels in a block of plates, respectively;

$g_{x, y}=w_{x, y} \cdot \rho \cdot f_{c h}$ - the mass flow rates through one channel of type $\mathrm{x}$ or $\mathrm{y}$. These

flow rates should satisfy equation $\Delta p_{x}=\Delta p_{y}$ and material balance:

$g_{x} \cdot n_{x}+g_{y} \cdot n_{y}=G_{b}$,

where $G_{b}$ - flow rate of the stream through the block of plates.

The principle of mixing plates in one heat exchanger gives the best results with symmetrical arrangement of passes $\left(\mathrm{X}_{1}=\mathrm{X}_{2}\right)$ and $\mathrm{G}_{\mathrm{b}}$ is equal to total flow rate of respective stream. The unsymmetrical arrangement $X_{1} \neq X_{2}$ is usually used when all channels are the same (any of the three available types). 
When the numbers of channels are determined, the numbers of plates calculated as:

$$
N_{p l}=\sum_{i=1}^{X_{1}}\left(n_{x 1 i}+n_{y 1 i}\right)+\sum_{j=1}^{X_{2}}\left(n_{x 2 i}+n_{y 2 i}\right)+1
$$

The total heat transfer area of PHE (two end plates not included), $\mathrm{m}^{2}$ :

$F_{\text {PHE }}=\left(N_{p l}-2\right) \cdot F_{p l}$,

where $F_{p l}$ - heat transfer area of one plate, $\mathrm{m}^{2}$.

The above algebraic equations (1)-(14) describe the relationship between variables which characterize PHE and heat transfer process in it. They can be regarded as a mathematical model of PHE, which solution enables to calculate pressure and temperature change of streams entering the heat exchanger. It is a problem of PHE rating (analysis).

The problem of PHE design (synthesis) require to find its characteristics (such as plate type, numbers of passes, numbers of plates with different corrugations) which will in the best way satisfy the required process conditions. Here the optimal design with pressure drop specification is considered, in a sense as described by Wang and Sunden (2003). The objective function is total heat transfer area of PHE with conditions that specified heat load $Q^{0}$ and allowable pressure drops for both streams $\Delta p_{1}{ }^{0}, \Delta p_{2}{ }^{0}$ must be satisfied. These conditions can be regarded as partial inequality constraints:

$$
Q \geq Q^{0} ; \quad \Delta p_{1} \leq \Delta p_{1}{ }^{0} ; \quad \Delta p_{2} \leq \Delta p_{2}{ }^{0}
$$

Analysis of relations (1) - (15) lets to conclude, that we have the mathematical problem of finding the minimal value for implicit nonlinear discrete/continues objective function with inequality constraints. It does not permit analytical solution without considerable simplifications. To solve it by numerical methods, the software is developed for IBM compatible PC. The mathematical model contains some parameters, namely coefficients and powers in empirical correlations that are not easily available.

\section{Identification of mathematical model parameters}

As a rule the empirical correlations for design of industrially manufactured PHEs are obtained during tests on such heat exchangers at specially developed test rigs. Such tests are made for every type of new developed plates and inter plate channels. The results are property of manufacturing company and usually not published.

Based on described above mathematical model it was developed the technique of numerical experiment that enables to identify model parameters by comparison with results obtained for the same conditions with the use of PHE calculation software, which is now available in internet for most of PHE manufacturers. The results for some plates manufactured by Alfa Laval presented in Table 1. The geometrical parameters of plates, for which correlations were acquired are given in Table 2.

The comparison of results obtained with our software to those of Alfa Laval free available software has shown good agreement (discrepancies not more then $4 \%$ on surface area). We should note, that obtained correlations and developed software can be used only for preliminary calculations, when optimizing PHEs or heat exchanger network. The final calculations when ordering the PHE must be performed by its manufacturer. 
Table 1. Parameters in correlations for some Alfa Laval PHEs ( $\mathrm{Re}>250)$

\begin{tabular}{|l|l|l|l|l|l|l|l|l|l|}
\hline $\begin{array}{c}\text { Plate } \\
\text { type }\end{array}$ & $\begin{array}{l}\text { Channel } \\
\text { type }\end{array}$ & $\mathrm{A}$ & $\mathrm{n}$ & $\mathrm{Re}$ & $\mathrm{B}$ & $\mathrm{m}$ & $\mathrm{Re}$ & $\mathrm{B}$ & $\mathrm{m}$ \\
\hline \multirow{3}{*}{$\mathrm{M} 3$} & $\mathrm{H}$ & 0.265 & 0.7 & $<520$ & 33.0 & 0.25 & $\geq 520$ & 10.7 & 0.07 \\
\cline { 2 - 10 } & $\mathrm{L}$ & 0.12 & 0.7 & $<1000$ & 18.8 & 0.33 & $\geq 1000$ & 8.8 & 0.22 \\
\cline { 2 - 10 } & $\mathrm{ML} / \mathrm{MH}^{1}$ & 0.18 & 0.7 & $<1000$ & 44.0 & 0.4 & $\geq 1000$ & 5.1 & 0.10 \\
\hline \multirow{3}{*}{ M6 } & $\mathrm{H}$ & 0.25 & 0.7 & $<1250$ & 10.0 & 0.2 & $\geq 1250$ & 2.4 & 0.0 \\
\cline { 2 - 10 } & $\mathrm{L}$ & 0.12 & 0.7 & $<1500$ & 5.1 & 0.3 & $\geq 1500$ & 1.7 & 0.15 \\
\cline { 2 - 10 } & $\mathrm{ML} / \mathrm{MH}$ & 0.165 & 0.7 & $<930$ & 9.3 & 0.3 & $\geq 930$ & 2.72 & 0.12 \\
\hline \multirow{3}{*}{ M6M } & $\mathrm{H}$ & 0.27 & 0.7 & $<1300$ & 11.7 & 0.13 & $\geq 1300$ & 4.55 & 0.0 \\
\cline { 2 - 10 } & $\mathrm{L}$ & 0.11 & 0.71 & $<2200$ & 4.23 & 0.23 & $\geq 2200$ & 1.88 & 0.12 \\
\cline { 2 - 10 } M10B & $\mathrm{ML} / \mathrm{MH}$ & 0.14 & 0.73 & $<2100$ & 5.61 & 0.16 & $\geq 2100$ & 1.41 & 0.0 \\
\cline { 2 - 10 } & $\mathrm{H}$ & 0.24 & 0.7 & $<2000$ & 11.1 & 0.15 & $\geq 2000$ & 3.5 & 0.0 \\
\cline { 2 - 10 } & $\mathrm{ML} / \mathrm{MH}$ & 0.11 & 0.7 & $<1500$ & 12 & 0.36 & $\geq 1500$ & 2.42 & 0.14 \\
\hline \multirow{3}{*}{ M15B } & $\mathrm{H}$ & 0.26 & 0.7 & $<2000$ & 5.84 & 0.05 & $\geq 2000$ & 5.84 & 0.05 \\
\cline { 2 - 10 } & $\mathrm{L}$ & 0.085 & 0.74 & $<2900$ & 5.2 & 0.28 & $\geq 2900$ & 1.57 & 0.13 \\
\cline { 2 - 9 } & $\mathrm{ML} / \mathrm{MH}$ & 0.13 & 0.74 & $<3500$ & 4.3 & 0.15 & $\geq 3500$ & 1.25 & 0.0 \\
\hline
\end{tabular}

Table 2. Geometrical parameters for some Alfa Laval PHE plates

\begin{tabular}{|c|c|c|c|c|c|c|c|}
\hline $\begin{array}{l}\text { Plate } \\
\text { type }\end{array}$ & $\begin{array}{c}\delta, \\
\mathrm{mm}\end{array}$ & $\begin{array}{c}d_{e}, \\
\mathrm{~mm}\end{array}$ & $\begin{array}{c}b, \\
\mathrm{~mm}\end{array}$ & $\begin{array}{c}\mathrm{F}_{\mathrm{pl}}, \\
\mathrm{m}^{2}\end{array}$ & $\begin{array}{c}D_{\text {connection }}, \\
\mathrm{mm}\end{array}$ & $\begin{array}{c}f_{c h} \cdot 10^{3}, \\
\mathrm{~m}^{2}\end{array}$ & $\begin{array}{c}L_{p}, \\
\mathrm{~mm}\end{array}$ \\
\hline $\mathrm{M} 3$ & 2.4 & 4.8 & 100 & 0.032 & 36 & 0.240 & 320 \\
\hline M6 & 2.0 & 4.0 & 216 & 0.15 & 50 & 0.432 & 694 \\
\hline M6M & 3.0 & 6.0 & 210 & 0.14 & 50 & 0.630 & 666 \\
\hline M10B & 2.5 & 5.0 & 334 & 0.24 & 100 & 0.835 & 719 \\
\hline M15B & 2.5 & 5.0 & 449 & 0.62 & 150 & 1.123 & 1381 \\
\hline
\end{tabular}

\section{Case study}

It is required to heat $5 \mathrm{~m}^{3} / \mathrm{h}$ of distillery wash fluid from 28 to $90{ }^{\circ} \mathrm{C}$ by hot water coming with temperature $95{ }^{\circ} \mathrm{C}$ and flow rate $15 \mathrm{~m}^{3} / \mathrm{h}$. The pressure of both fluids is 5 bar. Allowable pressure drop for hot stream 1.5 bar. For cold stream 1 bar. The properties of wash fluid are taken constant as follows: density $-978,4 \mathrm{~kg} / \mathrm{m}^{3}$; heat capacity $-3,18 \mathrm{~kJ} /(\mathrm{kg} \cdot \mathrm{K})$; cconductivity $-0.66 \mathrm{~W} /(\mathrm{kg} \cdot \mathrm{m})$. Dynamic viscosity at temperatures $t=25 ; 60 ; 90^{\circ} \mathrm{C}$ is taken as $\mu=19,5 ; 16,6 ; 9 \mathrm{cP}$.

The results of calculations for different passes numbers $X_{1}$ and $X_{2}$ and optimal for those passes plates arrangements are presented in Table 3 . The analysis show that the global optimum ( 38 plates) is achieved at $\mathrm{X}_{1}=2$ and $\mathrm{X}_{2}=4$ with all medium channels $(19 \mathrm{H}$ and $19 \mathrm{~L}$ plates in PHE). The closest other option ( 41 plates) is at $\mathrm{X}_{1}=\mathrm{X}_{2}=2$ with mixed

\footnotetext{
${ }^{1}$ In our study we neglected by small differences in ML and MH channels parameters
} 
Table 3. The influence of passes and plate arrangement on number of plates in M6M PHE

\begin{tabular}{|c|c|c|c|c|}
\hline \multirow{2}{*}{$\mathrm{X}_{2}$} & \multicolumn{4}{|c|}{$\mathrm{X}_{1}$} \\
\cline { 2 - 5 } & 1 & 2 & 3 & 4 \\
\hline 1 & $\frac{56 \text { plates }}{28 \mathrm{H} / 1 * 27 \mathrm{H}}$ & $2 * \frac{235 \text { plates }}{59 \mathrm{H} / 1 * 116 \mathrm{H}}$ & $3 * 26 \mathrm{H} / 1 * 78 \mathrm{H}$ & $4 * 23 \mathrm{H} / 1 * 91 \mathrm{H}$ \\
\hline 2 & $\underline{72 \text { plates }}$ & $\underline{41 \text { plates }}$ & $\underline{60 \text { plates }}$ & $\frac{64 \text { plates }}{4 * 8 \mathrm{~L} /}$ \\
& $1 * 35 \mathrm{H} / 2 * 18 \mathrm{H}$ & $2 *(7 \mathrm{H}+3 * \mathrm{ML}) /$ & $3 * 10 \mathrm{MH} /$ & $2 * 15 \mathrm{~L}+1 * 16 \mathrm{~L}$ \\
\hline 3 & $\underline{44 \text { plates }}$ & $\underline{49 \text { plates }}$ & $\underline{43 \text { plates }}$ & $\underline{50 \text { plates }}$ \\
& $1 * 21 \mathrm{H} /$ & $3 * 8 \mathrm{ML}$ & $3 *(4 \mathrm{MH}+3 * \mathrm{~L}) /$ & $3 * 6 \mathrm{~L}+1 * 7 \mathrm{~L} /$ \\
& $2 * 7 \mathrm{H}+1 * 8 \mathrm{H}$ & $\underline{38 \text { plates }}$ & $\underline{44 \mathrm{ML}+3 * \mathrm{~L})}$ & $3 * 8 \mathrm{~L}$ \\
\hline 4 & $\underline{63 \text { plates }}$ & $1 * 9 \mathrm{MH} /$ & $3 * 7 \mathrm{~L} /$ & $3 * 6 \mathrm{plates}$ \\
& $1 * 7 \mathrm{ML}+3 * 8 \mathrm{ML}$ & $1 * 4 \mathrm{ML}+3 * 5 \mathrm{ML}$ & $2 * 5 \mathrm{~L}+2 * 6 \mathrm{~L}$ & $4 * 6 \mathrm{~L}$ \\
\hline
\end{tabular}

channel arrangement in one pass. If we would have only one plate type in PHE, the minimal number of plates would be 44 for both $\mathrm{H}$ and $\mathrm{L}$ plates, or $15 \%$ higher than with mixed channels.

\section{Conclusions}

The algorithm and software for computer aided design of multi-pass PHE assembled with plates of different corrugation patterns is developed. The model parameters corresponding to some industrially manufactured plates are obtained. The examples of calculation results for case study show the possibility with such method to obtain optimal solutions with exact satisfaction of constraints for total heat load and pressure drop of one stream. It gives the considerable reduction in heat transfer surface area of PHE.

\section{Acknowledgements}

The financial support of EC Project ECOPHOS (contract № INCO-CT-2005-013359) is sincerely acknowledged.

\section{References}

O. Arsenyeva, L. Tovazhnyansky, P. Kapustenko, G. Khavin, 2009, Mathematical Modelling and Optimal Design of Plate-and-Frame Heat Exchangers. Chemical Engineering Transactions, 18: 791-796.

A. Gogenko, O. Anipko, O. Arsenyeva, P. Kapustenko, 2007, Accounting for fouling in plate heat exchanger design. Chemical Engineering Transactions, 12: 207-213.

J. Hesselgreaves, 2001, Compact Heat Exchangers. Selection, Design and Operation. Elsevier, Amsterdam.

L. Tovazshnyansky, P. Kapustenko, G. Khavin, O. Arsenyeva., 2004, PHEs in Industry. NTU KhPI, Kharkiv (in Russian).

L. Wang, B. Sunden, R. Manglik , 2007, PHEs. Design, Applications and Performance. WIT Press, Southhampton.

L. Wang, B. Sunden, 2003, Optimal design of PHEs with and without pressure drop specifications. Applied Thermal Engineering. 23, 295-311. 